\title{
Laser-based Geometric Modeling using Cooperative Multiple Mobile Robots
}

\author{
Ryo Kurazume, Yusuke Noda, Yukihiro Tobata, Kai Lingemann, Yumi Iwashita and Tsutomu Hasegawa
}

\begin{abstract}
In order to construct three-dimensional shape models of large-scale architectural structures using a laser range finder, a number of range images are taken from various viewpoints. These images are aligned using post-processing procedures such as the ICP algorithm. However, in general, before applying the ICP algorithm, these range images must be aligned roughly by a human operator in order to converge to precise positions. The present paper proposes a new modeling system using a group of multiple robots and an on-board laser range finder. Each measurement position is identified by a highly precise positioning technique called Cooperative Positioning System (CPS), which utilizes the characteristics of the multiple-robot system. Thus, the proposed system can construct 3D shapes of large-scale architectural structures without any post-processing procedure or manual registration. ICP is applied optionally for a subsequent refinement of the model. Measurement experiments in unknown and large indoor/outdoor environments are carried out successfully using the newly developed measurement system consisting of three mobile robots named CPS-V. Generating a model of Dazaifu Tenmangu, a famous cultural heritage, for its digital archive completes the paper.
\end{abstract}

\section{INTRODUCTION}

For constructing 3D models of large-scale architectural structures using laser range finders, a number of range images are taken from various viewpoints. These images are aligned using post-processing procedures, such as the ICP algorithm [1], [2]. This is necessary, because a laser range finder cannot cover the entire area at one time due to the limitation of measurable distance and occlusion problems. After post-processing procedures, the range images described in the sensor coordinate system are transformed to the global coordinate system, and the entire shape of the architecture is obtained. However, in general, when sufficiently exact scan pose estimates are not given, a human operator registers the range images to correct positions roughly before applying the ICP method in order to ensure that the images converge to proper poses. This procedure is quite laborious and timeconsuming and is considered to be a significant obstacle for developing an automatic 3D laser measurement system. In addition, all of the images must contain sufficient feature

R. Kurazume, Y. Noda, Y. Tobata, Y. Iwashita, and T. Hasegawa are with Graduate Faculty of Information Science and Electrical Engineering, Kyushu University, 744 Motooka, Nishi-ku, Fukuoka, Japan kurazume@is.kyushu-u.ac.jp

Y. Noda is with Graduate School of Information Science and Electrical Engineering, Kyushu University, 744 Motooka, Nishi-ku, Fukuoka, 8190395, Japan

Y. Tobata is with LinX Corporation, 1-13-11, Eda-nishi, Aoba-ku, Yokohama, 225-0014, Japan

Kai Lingemann is with University of Osnabrück, Albrechtstr. 28, 31/506, D-49069, Osnabrück, Germany shapes and must sufficiently overlap each other, which requires dense scanning from a number of positions, in order to register range images precisely by the ICP algorithm.

Another approach that requires no post-processing procedures such as the ICP algorithm can also be considered. It involves the precise identification of the position of the range sensor at each measurement. Since this method can obtain the transformation matrix from the sensor specific coordinate system to the global coordinate system, local range images are converted directly into the global coordinate system with a simple transformation calculation. As an example of this approach, several systems that utilize GPS [3], [4] to determine the position of the range sensor have been proposed. However, special instruments and techniques, such as a Real-time Kinematic (RTK) system or the Virtual Reference Station (VRS) method, are required in order to achieve highly precise position identification by current GPS. Moreover, GPS cannot be used unless a sufficient number of satellites can be accessed which leads to problems in, for example, narrow structures, forests, or indoor environments.

The present paper proposes a new 3D measurement system for large-scale architectural structures using a group of mobile robots and an on-board laser range finder. The proposed system uses the Cooperative Positioning System (CPS) [5], [6] for multiple robots, which has been proposed as a highly precise position identification technique for mobile robots, and an automatic measurement system for large-scale architectural structures is realized by combining highly precise position identification by CPS and an onboard laser range finder. This system can construct a 3D model of a large-scale architectural structure without any post-processing procedure such as the ICP algorithm or registration by a human operator. In addition, it is possible to register range images even if the number of measurements is few and only sparse range images, e.g., range images containing insufficient feature shapes or overlappping regions, are available. It is also possible to construct $3 \mathrm{D}$ model in environments where GPS is not available, such as inside structures or in an indoor environment.

The proposed system is related to the simultaneous localization and mapping (SLAM) method [7]-[14], which is attracting a great deal of attention in the robotics community. As shown in Section III.B, the 3D model obtained by CPS is refined by applying ICP. Obviously, the refined measurement position from ICP can also be fed back to the CPS positioning system. This closed loop control will increase the accuracy of both the 3D model and robot position.

This paper introduces measurement experiments in un- 
known and large indoor/outdoor environments using the newly developed measurement system consisting of three mobile robots named CPS-V. In addition, as an example of applications of the proposed system, we introduce the digital archive experiments of Dazaifu Tenmangu, which is Japanese famous cultural heritage and one of the important cultural properties of Japan.

\section{Cooperative Positioning System (CPS)}

Let us consider the system in which a mobile robot equipped with an on-board laser range finder moves around a measurement target and scans the target from several different positions. If all of the measurement positions are identified with high accuracy, the range data acquired at each position can be converted to the global coordinate system by a simple coordinate transformation calculation.

Several position identification methods have been proposed, and these methods can be classified into four categories:

1) Integrate sensor output from internal sensors such as wheel encoders or acceleration sensors.

2) Observe external landmarks by external sensors such as laser range finders or cameras.

3) Use the Global Positioning System (GPS).

4) The simultaneous localization and mapping (SLAM) method

Method (1) is referred to as odometry, or dead reckoning, and is a commonly used positioning technique, especially for wheeled vehicles. However, there are drawbacks associated with this method. For example, the accuracy of position identification in uneven terrain is quite low due to slippage of the wheels, and 3D positioning including elevation is impossible. Method (2) has high accuracy if landmarks placed on the moving path can be measured precisely. However, landmarks must be placed beforehand along the moving path, and a precise map of these landmarks must be available. Therefore, method (2) cannot be used in an unknown environment. Method (3) can be considered as a special case of method (2) and is becoming very popular, especially for field robots. However, this method also has several drawbacks. For example, active fields of robots are limited to an outdoor environment with an unobstructed view of satellites, and the accuracy is currently not so high, unless special techniques described in Section I are used. Method (4) is based on the matching of sequentially observed local features acquired by external sensors such as laser range finders or cameras. When considering the features as landmarks, this constitutes a general case of method (2). Though this method is a prospective technique for precise position identification in unknown and unstructured environment, precise and sufficient information between consequtive observations must be acquired through dense measuremnts.

To overcome these limitations of position identification problems and realize accurate positioning of mobile robots, Kurazume et al. proposed the Cooperative Positioning System (CPS) [5]. In this system, multiple robots with highly precise measurement devices of mutual positions are controlled cooperatively, and unsurpassed positioning accuracy, as compared to conventional positioning techniques, is realized, even in unknown and uneven environments.

The basic principle of CPS is as follows: We divide the robots into group A and group B. Group A remains stationary and acts as a landmark while group B moves. Group B then stops and acts as a landmark for group A. This alternating behavior is repeated until the target position is reached. By using the concept of "portable landmarks", CPS has a far lower accumulation of positioning error than dead reckoning and can work in three dimensions, which is not possible by ordinary dead reckoning. In addition, CPS can be used in an unknown environment, since there is no need to place landmarks beforehand.

An example of CPS is shown in Fig.1. This example is for a robot system consisting of a parent robot with a sensing device such as a laser range finder, and two child robots. The sensing device can measure the relative positions of the child robots from the parent robot. First, we assume that the initial position of the parent robot is measured or defined beforehand.

(1) Child robots 1 and 2 are moved and stopped.

(2) The parent robot measures the distance, azimuth, and elevation angles to child robot 1 and identifies the position of child robot 1 .

(3) The position of child robot 2 is identified in the same manner as Step 2.

(4) The parent robot moves and stops. The distances, azimuth, and elevation angles to child robots 1 and 2 are then measured, and the position of the parent robot is calculated using the triangular surveying technique.

(5) Repeat Steps 1 through 4 until the target position is reached.

Though the principle of CPS is simple, the position calculation which suppresses the error accumulation is rather complicated [6]. In CPS, though the accuracy is quite high, the measurement errors are gradually accumulating and the characteristics of errors depend on the moving histories of the robots. To minimize the error accumulation by taking the moving histories into account, we proposed the non-linear least squared method based on the sequential estimation of error covariance matrices. The performance of CPS has been tested through many experiments in a variety of environments such as an unpaved outdoor environment with steep sloops, and confirmed that, compared to odometry, a highly precise robot tracking is achieved [6].

\section{Construction OF A 3D EnVironmental MAP BY MULTIPLE ROBOTS}

This section proposes a new measurement system for precise construction of a 3D environmental map by combining CPS for multiple robots and a laser range finder. In this system, mobile robots move around a large-scale target and scan the target by an on-board 3D laser range finder from several viewpoints. Each measurement position is precisely identified by CPS using a parent robot and two child robots. 


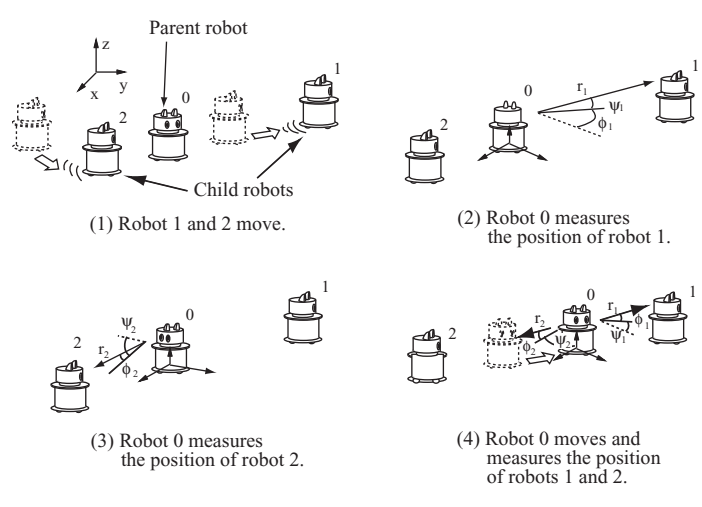

Fig. 1. Cooperative Positioning System (CPS)

First, we introduce the fifth CPS machine model, called CPS$\mathrm{V}$, which is equipped with a $2 \mathrm{D}$ laser range finder and a scanning mechanism, and show the experimental results for the construction of indoor and outdoor environmental maps by CPS-V.

\section{A. Fifth CPS machine model (CPS-V)}

Figure 2 shows the fifth CPS machine model, CPS-V. This system consists of a parent robot (P-cle, Parent mobile unit, Fig.3(a)) and two child robots (HPI Japan, Fig.3(b)). The parent robot is equipped with an on-board 2D laser range finder (LMS 200, Sick), a high-precision two-axis attitude sensor (MD900T, Applied Geomagnetics), and a total station for surveying (GTS-825A, TOPCON Ltd.)(Tab. I), which is used to measure the relative positions of the child robots. Even if the body is tilted on a slope, the body inclination is compensated by an attitude sensor, and precise positions of the robots is possible. The 2D laser range finder can acquire 2-dimensional slit-like range data within the range of $80 \mathrm{~m}$ and 180 degrees, as shown in Tab. II. The parent robot has a rotating table on the top body, and by rotating the table around the vertical axis while scanning using the 2D laser range finder, $360^{\circ} 3 \mathrm{D}$ range images are acquired from the parent robot in 37.8 seconds.

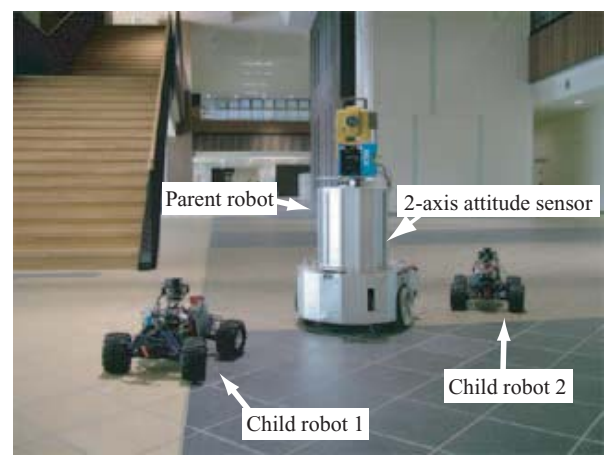

Fig. 2. Fifth CPS machine model, CPS-V

\section{B. Construction experiment of the indoor environmental map}

1) Initial modeling by $C P S-V$ : Experiments for constructing 3D maps are carried out using CPS-V in an indoor environment. In this experiments, the parent robot moved,
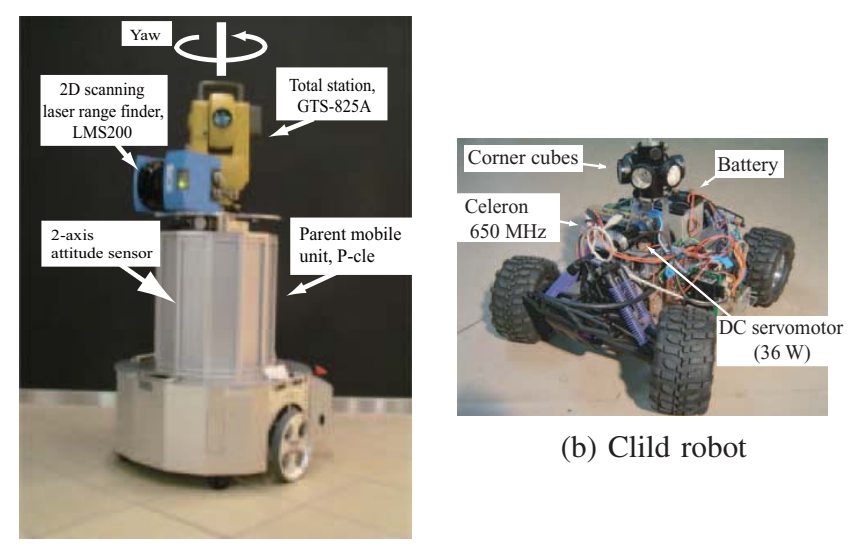

(b) Clild robot (a) Parent robot

Fig. 3. Robots with 3D laser measurement using the rotation table around the yaw axis

TABLE I

Specifications of THE total Station, GTS-825A

\begin{tabular}{l|r}
\hline \multicolumn{2}{c}{ GTS-825A (TOPCON Ltd.) } \\
\hline Range & $1.3 \sim 2,200[\mathrm{~m}]$ \\
Resolution (distance) & $0.2[\mathrm{~mm}]$ \\
Resolution (angle) & $3 ”$ \\
Precision (distance) & $\pm 2+2 \mathrm{ppm}[\mathrm{mm}]$ \\
Precision (angle) & $\pm 5 ”$ \\
\hline
\end{tabular}

stopped, and identified its position 22 times. The positions of robots are currently determined by an operator so that the number of movements of the child robots becomes as small as possible. After identifing the position, the parent robot captured 3D range images at each stationary position by rotating the on-board 2D laser range finder around the vertical axis. The obtained range images are transformed into the global coordinate system by simple coordinate transformation calculation using the position measured by CPS. No post-processing procedure is applied so far. The positioning error of CPS is $0.22 \mathrm{~m}$, compared to the total moving distance of the parent robot of $93.9 \mathrm{~m}(0.24 \%$ of the distance traveled). The error is measured using reference corner cubes around the starting position. Figure 7(a) depicts the difference in measured positions of the corner indicated in Fig.4. From this figure, we can estimate that the modeling error is about $220 \mathrm{~mm}$. Figures 5 and 6 shows the total view and some images of the obtained 3D map.

2) Optional refinement via ICP: If the scans do display enough overlap, an additional step for finalizing the map is applied: The obtained 3D model is refined automatically using a version of the Iterative Closest Points (ICP) algorithm. The implemented method registers range images against a so-called metascan, i.e., the union of the previously acquired and registered scans, and the 3D model is grobally optimized.

A significant speed-up is achieved by organizing the data - as well as reduced data sets based on an octree representation of the scans - in a $k$-D tree structure. These trees are furthermore cached, exploiting a buffer for leaf nodes; the buffer is used to start backtracking in subsequent searches.Therefore, the iterative structure of ICP is exploited for reducing computation costs. By using these mechanisms, the registration of the whole 22 scans of this experiment took 
TABLE II

SPECIFICATION OF THE LASER RANGE FINDER, LMS200

\begin{tabular}{l|r}
\hline \multicolumn{2}{c}{ LMS 200 (SICK Corp.) } \\
\hline Range & $80[\mathrm{~m}]$ \\
Field of view & $180^{\circ}$ \\
Resolution (distance) & $10[\mathrm{~mm}]$ \\
Resolution (angle) & $0.5^{\circ}$ \\
\hline
\end{tabular}

9.3 seconds only.

Figure 7(b), showing the registration results after applying ICP, demonstrates the optimal cooperation of both algorithms: The first step, alignment by CPS, yields a consistent and mostly accurate map - without human intervention -, which guarantees that the scans' estimated poses are sufficiently exact for a safe and stable refinement by ICP. This way, we were able to reduce the modeling error to less than $8.5 \mathrm{~mm}$. In fact, the combined work of these two methods provided such accurate results that an execution of global 3D optimizationdid not noticeably change the output in our experiment.

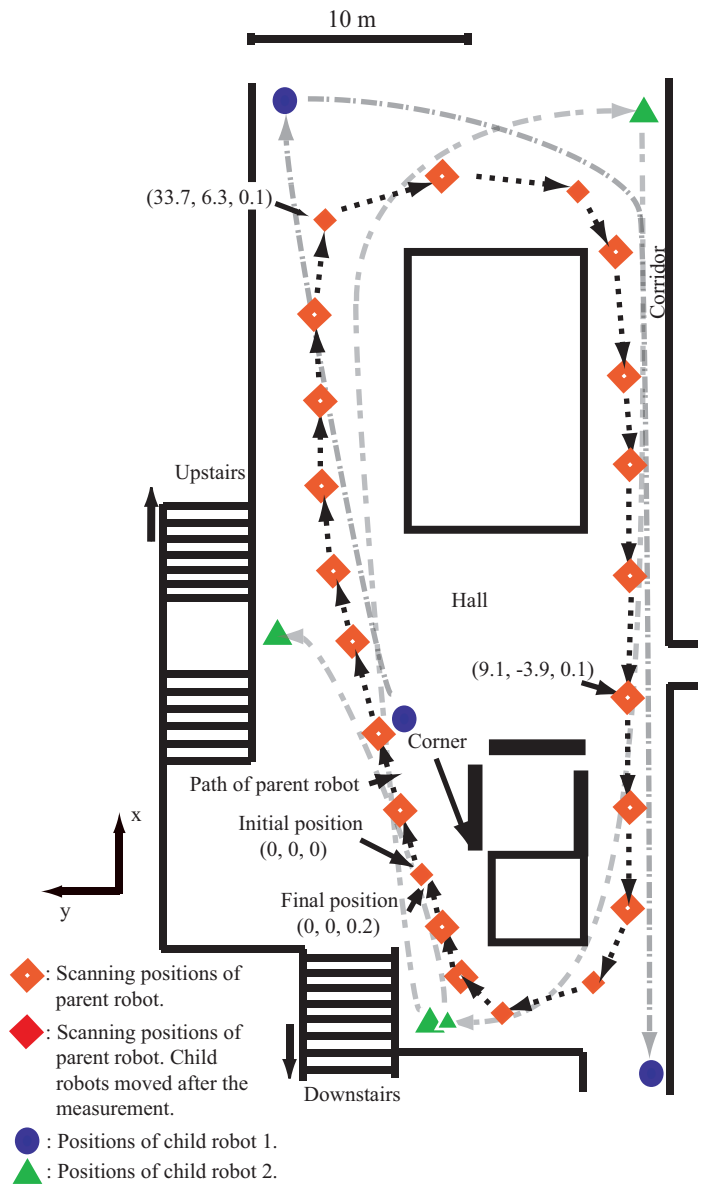

Fig. 4. Path of the parent robot

\section{Construction experiment to obtain a large-scale outdoor environmental map}

Next, we performed a construction experiment to obtain 3D environmental maps of large-scale architectural structures. We measured the outer walls of a building from

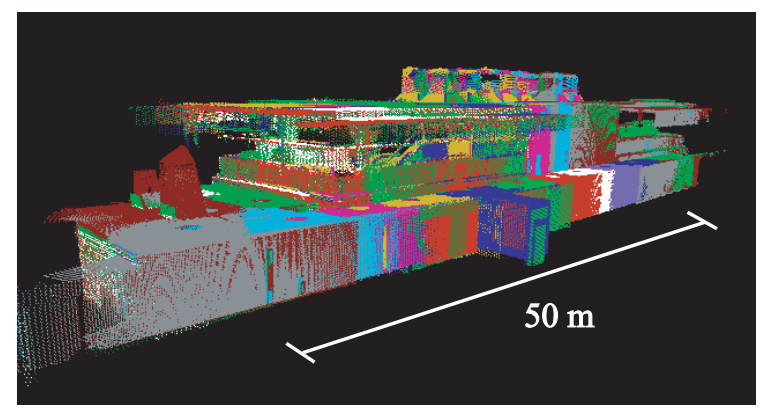

Fig. 5. 3D map of the indoor environment

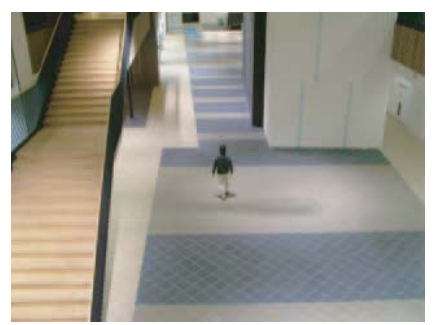

(a1)

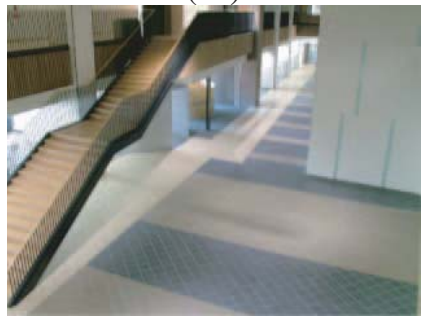

(b1)

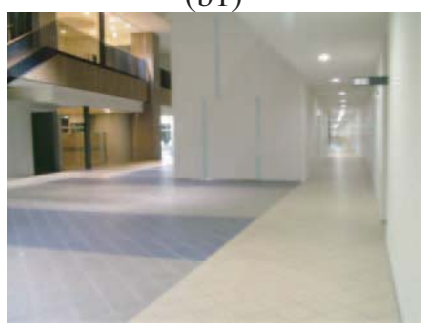

(c1)

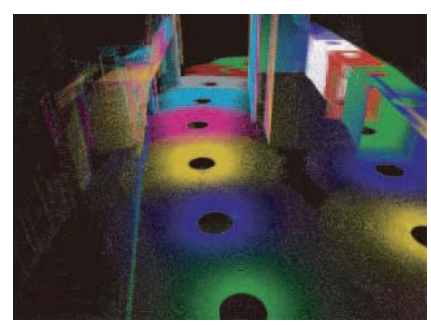

(a2)

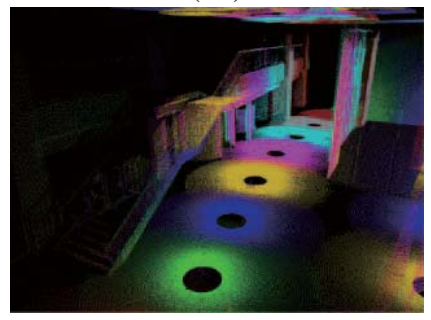

(b2)

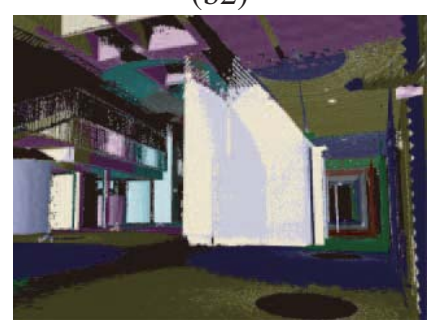

(c2)
Fig. 6. 3D model of the indoor environment

13 points in the outdoor environment and constructed 3D models of the building. The path of the parent robot and the obtained maps are shown in Figs.8, 9, and 10, respectively. In this experiment, we used a small number of measurements from sparse viewpoints. Neither dense range data nor sufficiently overlapped regions, which are indispensable for applying the ICP algorithm, were available. Based on this experiment, we concluded that it is possible to construct 3D maps of simple-shaped but large-scale architectural structures using the proposed system with only a few scans. The positioning error in this experiment is $0.63 \mathrm{~m}$, compared to the total travel distance of the parent robot of $147.7 \mathrm{~m}$, which is $0.43 \%$ of the distance traveled.

\section{ApPliCATION FOR THE Digital archive of LARGE-SCALE CULTURAL HERITAGES}

This section introduces an example of the application of the proposed system for the digital archive of a large-scale 


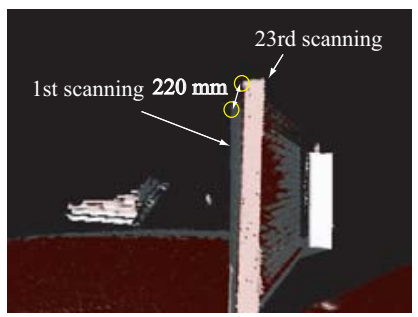

(a) Modeling error by CPS

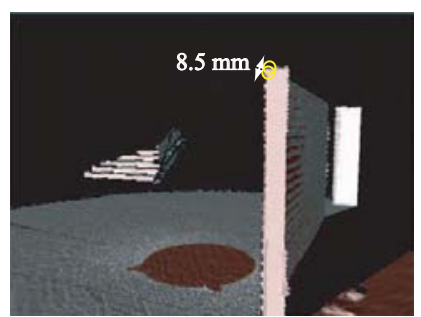

(b) After applying ICP

Fig. 7. Comparison of positioning error

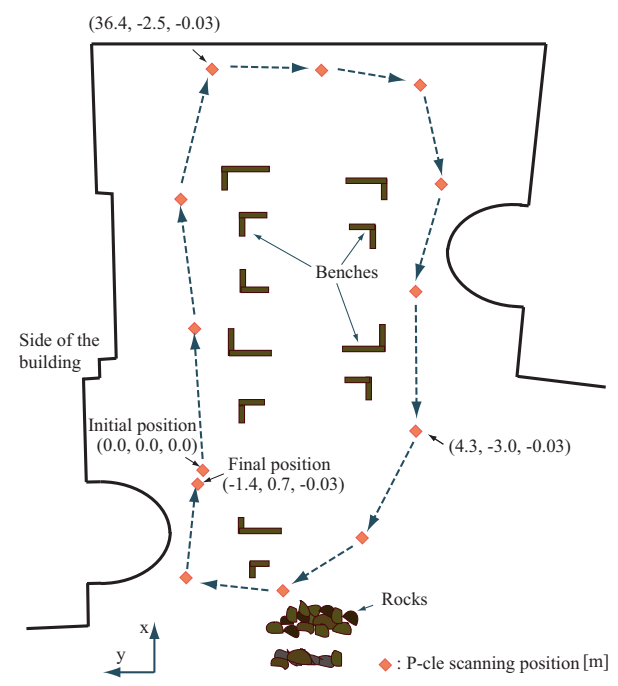

Fig. 8. Path of the parent robot in the outdoor environment

Japanese cultural heritage.

Dazaifu Tenmangu (Dazaifu Shrine) (Fig.11) in Fukuoka, Japan, was established in 919 by Michizane Sugawara who is known as the god of leaning. The main shrine of the Dazaifu Tenmangu was built in 1591 and is registered as an important cultural property of Japan. The size of the main shrine and the yard are about $250 \mathrm{~m} \times 100 \mathrm{~m}$.

In August 2008, we successfully carried out the 3D digital archive experiment of the main shrine of Dazaifu Tenmangu and the vast garden by the robot system proposed in this paper. Figure 14 shows the total view of Dazaifu Tenmangu

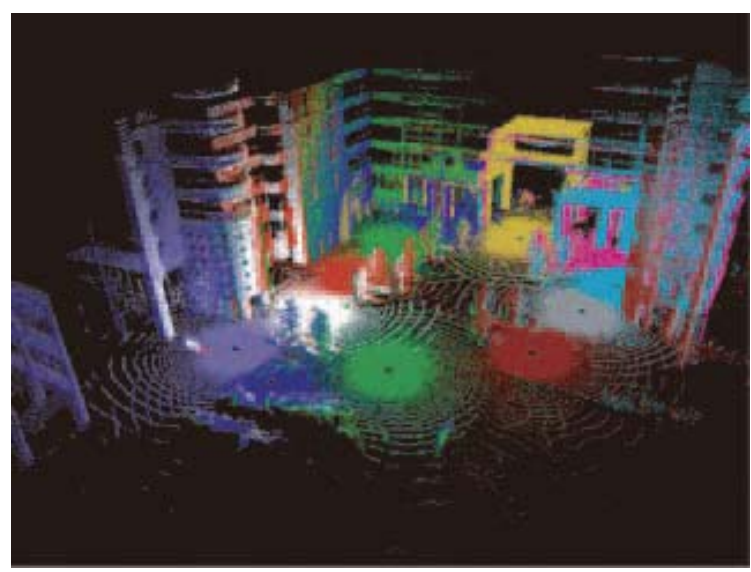

Fig. 9. 3D map of buildings in the outdoor environment
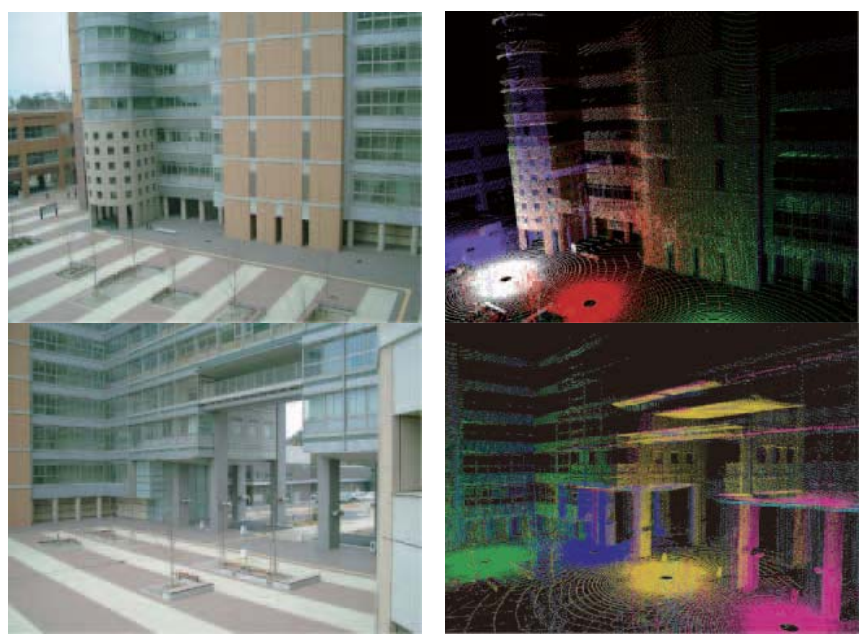

Fig. 10. 3D model of buildings in the outdoor environment

and Figs. 12 and 13 show the 3D model of the main shrine and the garden including three famous bridges over the pond named "Shinji Ike". The parent robot moved and measured the Dazaifu Tenmangu from 76 places in the inside and outside of the main shrine and the garden.
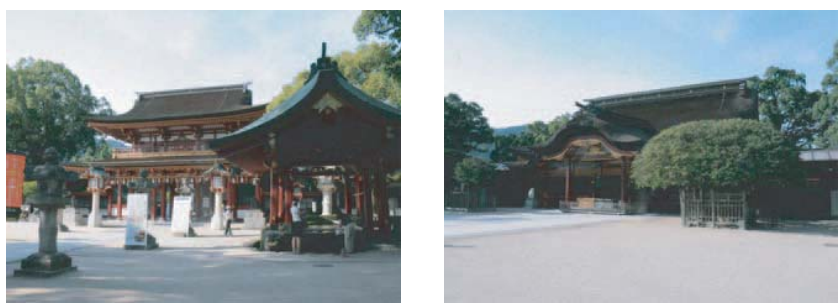

Fig. 11. Dazaifu Tenmangu (Dazaifu Shrine)

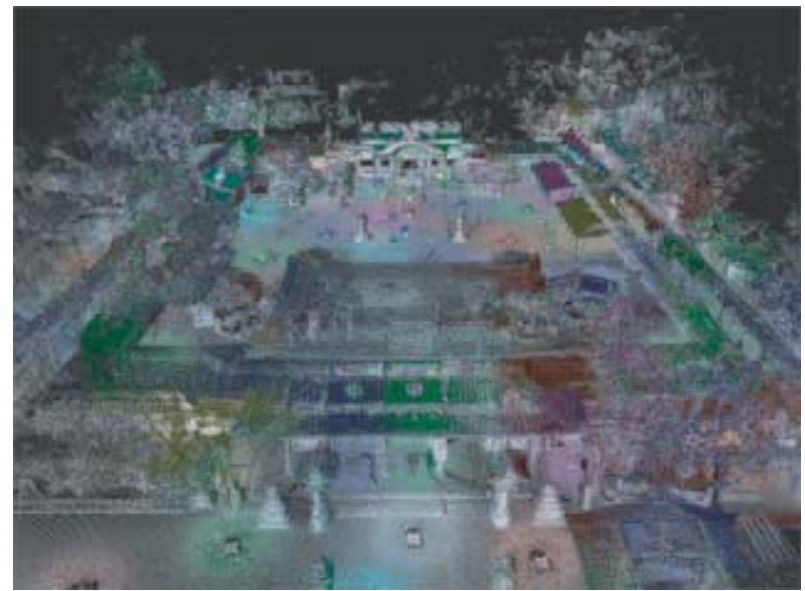

Fig. 12. 3D model of the main shrine of Dazaifu Tenmangu

\section{Conclusions}

A new 3D measurement system was proposed for largescale architectural structures using a precise positioning system called the Cooperative Positioning System (CPS) involving multiple robots and an on-board laser range finder. The proposed system enables to create a 3D model without post-processing procedures, and can be applied to sparse 


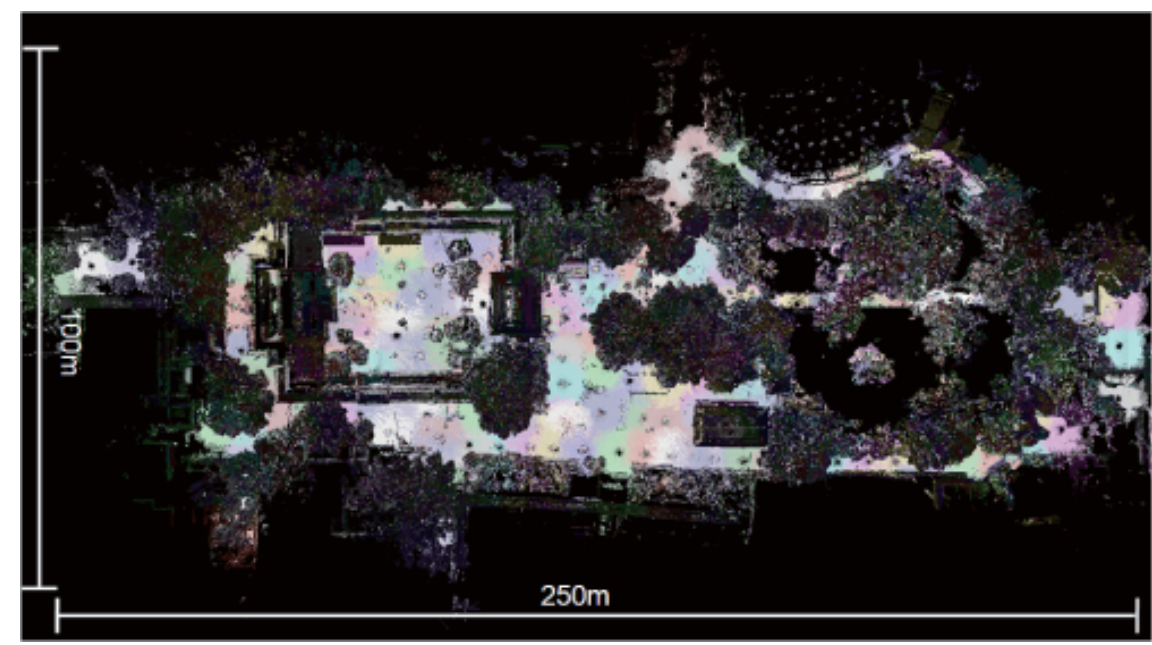

Fig. 14. Total view of the 3D model of Dazaifu Tenmangu

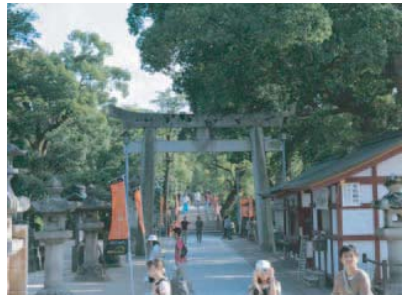

(a1)

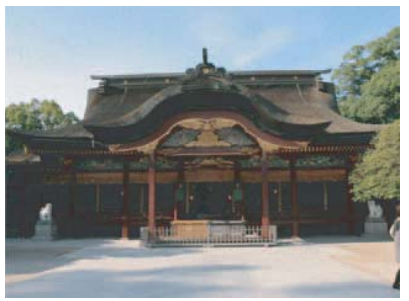

(b1)

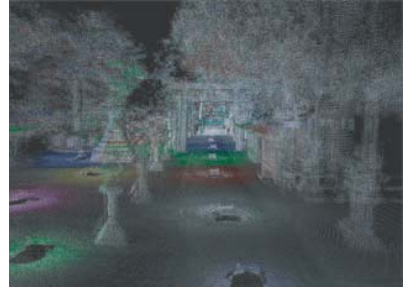

(a2)

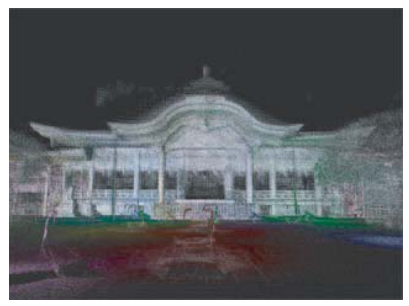

(b2)

Fig. 13. Constructed 3D model of Dazaifu Tenmangu

range measurements. ICP can be applied optionally for a subsequent refinement of the model. Therefore, a fully automatic 3D measurement system for large-scale architectural structures, such as large, culturally important structures, will be realized based on the proposed system. Measurement experiments in unknown and large indoor/outdoor environments were successfully carried out using the newly developed multiple-robot system, called CPS-V. Generating a model of Dazaifu Tenmangu, a famous cultural heritage, for its digital archive completed the paper.

In the future, we intend to investigate the development of an automatic 3D laser measurement system that will automatically select optimum measurement points. The positioning accuracy of CPS can also be further improved by back-projection, that is, the observation of some stable landmarks before and after the movement and the correction of each measurement position by back-projecting the errors. In addition, the combination of CPS and the ICP method not only for the refinement of the model but also the improvement of the accurate positioning will be considered.

\section{REFERENCES}

[1] P. J. Besl and N. D. McKay, "A method for registration of 3-d shapes," IEEE Transactions on Pattern Analysis and Machine Intelligence, vol. 2, no. 14, pp. 239-256, 1992.

[2] Y. Chen and G. Medioni, "Object modelling by registration of multiple range images," Image and Vision Computing, vol. 3, no. 10, pp. 145$155,1992$.

[3] H. Zhao and R. Shibasaki, "Reconstructing a textured cad model of an urban environment using vehicle-borne lase range scanners and line cameras," Machine Vision and Applications, vol. 14, pp. 35-41, 2003.

[4] K. Ohno, T. Tsubouchi, and S. Yuta, "Outdoor map building based on odometory and rtk-gps positioning fusion," Proc. IEEE International Conference on Robotics and Automation, pp. 684-690, April 2004

[5] R. Kurazume, S. Nagata, and S. Hirose, "Cooperative positioning with multiple robots," Proc. IEEE Int. Conf. on Robotics and Automation, vol. 2, pp. 1250-1257, 1994.

[6] R. Kurazume and S. Hirose, "An experimental study of a cooperative positioning system," Autonomous Robots, vol. 8, no. 1, pp. 43-52, 2000.

[7] S. Thrun, "A probabilistic online mapping algorithm for teams of mobile robots," International Journal of Robotics Research, vol. 20, no. 5, pp. 335-363, 2001.

[8] A. Nüchter, H. Surmann, K. Lingemann, J. Hertzberg, and S. Thrun, "6d slam with an application in autonomous mine mapping," Proc. IEEE International Conference on Robotics and Automation, pp. 1998-2003, 2004.

[9] J. Weingarten and R. Siegwart, "Ekf-based 3d slam for structured environment reconstruction," Proc. IEEE/RSJ International Conference on Intelligent Robots and System,, pp. 2089-2094, 2005.

[10] D. M. Cole and P. M. Newman, "Using laser range data for $3 \mathrm{~d}$ slam in outoor environment," Proc. IEEE International Conference on Robotics and Automation, pp. 1556-1563, 2006.

[11] S. Thrun and M. Montemerlo, "The graph slam algorithm with applications to large-scale mapping of urban structures," International Journal of Robotics Research, vol. 25, no. 5-6, pp. 403-429, 2006.

[12] A. Howard, "Multi-robot simultaneous localization and mapping using particle filters," International Journal of Robotics Research, vol. 25, no. 125, pp. 1243-1256, 2006.

[13] R. Triebel, P. Pfaff, and W. Burgard, "Multi-level surface maps for outdoor terrain mapping and loop closing," International Conference on Intelligent Robots and Systems(IROS), pp. 2276-2282, 2006.

[14] M. D. Marco, A. Garulli, A. Giannitrapani, and A. Vicino, "Simultaneous localization and map building for a team of cooperating robots: a set membership approach," IEEE Transaction on Robotics and Automation, vol. 19, no. 2, pp. 1243-1256, 2003. 